\title{
Indoor Coverage Prediction and Optimization for UMTS Macro Cells
}

\author{
Wolfgang Karner, Alexander Paier, Markus Rupp \\ Institute of Communications and Radio-Frequency Engineering \\ Vienna University of Technology, Austria \\ Gusshausstrasse 25/389, A-1040 Vienna, Austria \\ Email: \{wkarner, apaier, mrupp\}@nt.tuwien.ac.at
}

\begin{abstract}
As most of the time mobile devices are used inside buildings, it is of particular interest for mobile network operators to cover indoor areas with their services. In order to reach satisfying indoor coverage without having too much extra costs for e.g. additional cell sites, optimum network planning has to be performed with the aid of coverage prediction by using propagation models. In this work we compare eight empirical and one deterministic model (ray tracing) by their usability and general validity for predicting the indoor coverage in UMTS macro cells. Furthermore, the accuracy of the models is shown via a comparison to measurements inside an office building in a live UMTS network in the city center of Vienna, Austria. It was found that despite the high effort for the usage, the deterministic model could not reach higher accuracy than the empirical models. Additionally, in this work we investigate improvements of the indoor coverage in UMTS macro cells by optimizing various base station antenna parameters.
\end{abstract}

\section{INTRODUCTION}

Nowadays, for most of the people using mobile phones is a part of their everyday life. Everybody wants to be reachable at any time and at any location. Since we spend a majority of our time inside buildings, it is important for the mobile communication operators to cover indoor areas.

Especially in UMTS networks with macro cells it is very difficult to reach sufficient indoor coverage due to the higher carrier frequency and the higher required throughput compared to GSM. By installing extra cells and tolerating high transmit power per link, it may be possible to reach the required coverage. However, network operators also try to avoid overdimensioning of their networks as this results in high costs for the additional cell sites or in a waste of radio resources. Thus, in order to reach a satisfying indoor coverage without great expenses, it is necessary to have a prediction of the effective coverage.

Particularly within buildings in urban areas the prediction of the received power levels is a very complex task due to the influence of the surrounding area, the vegetation and for example the high attenuation of reinforced concrete walls or metallized glass facades. Since the accurate computation of the received power is not possible due to the high complexity, various models are used to predict the indoor coverage.

Lots of research has already been accomplished for modeling the outdoor-indoor propagation. In this document we are comparing eight empirical models [1]-[8] and one simulation tool 'Wireless InSite' from REMCOM [9] which is based on a deterministic model (ray tracing). We are evaluating the different models for calculating the received power levels within buildings in UMTS macro cells. The accuracy of the models is evaluated by comparing the predictions of the models with the results out of measurements performed in a live UMTS network in an office building in the city center of Vienna, Austria. Furthermore, apart from the evaluation of the accuracy, we are comparing the mentioned empirical or deterministic models due to their universal validity and usability which is very important particularly for network operators.

An improvement of the UMTS coverage is expected e.g. by increasing the transmitted $\mathrm{CPICH}$ (Common Pilot Channel) and the maximum link power levels. Due to the interference limitation in CDMA systems, this results in decreasing capacity in the whole network. Thus, in order to reach a high level of indoor coverage in macro cells not only the transmitted power levels should be considered, but also other radio network parameters which are influencing the path loss between the base station and the mobile device. In this work we show results for the optimization of the coverage in one office building by varying the height and position of the base station antenna, the antenna down-tilt and the antenna pattern.

This document is organized as follows. In Section II the models for predicting the outdoor-indoor propagation are compared by their characteristics and usability, where II(A) is dedicated to eight empirical models and in $\mathrm{II}(\mathrm{B})$ there is a brief description of the deterministic model and the setup of the corresponding simulation tool ('Wireless InSite'). The investigated scenario for comparing the measurements with the modeling is described in Section III and in Section IV the results of the evaluation concerning the accuracy of the models are shown. Section $\mathrm{V}$ presents some ideas for the optimization of the indoor coverage and finally, Section VI provides a summary and conclusions.

\section{MODELS FOR OUTDOOR-INDOOR RADIO PROPAGATION PREDICTION}

The models for calculating the propagation loss can be divided into empirical and deterministic models. In case of empirical models analytical equations are designed out of extensive field measurements. Deterministic models are resulting from theory rather than from experience. One type of deterministic models 
are the ray-optical models, which are tracing the rays over their reflections at and via their transmissions through objects in the propagation path up to the desired receiving point.

\section{A. Empirical Models}

Empirical models usually are very simple and efficient to use, meaning that no accurate data of the propagation area is necessary and that they are not time consuming in the computation of the received power level. As input for the empirical models only a few empirical and some other parameters are required. Empirical parameters e.g. represent the penetration loss of the building walls. The other parameters are parameters like the distance between the base station antenna and the external wall or the indoor receiving point, the height above the street level, the angle of incidence, the number of internal walls or the frequency. The more parameters are used in one model, the better the model can be adapted to different scenarios. In order to achieve best possible results, all the empirical parameters have to be calibrated by experiments for a new propagation scenario.

The disadvantage of the empirical models is the limited accuracy and furthermore, only a calculation of the received power level is possible up to now.

In this work we compare eight empirical models for radio propagation into buildings. Beginning with a very simple model from R. Hoppe, presented in [1], we have also been evaluating the model developed by Z. Liu [2] and a further development from that which is described in [3]. Our comparison also includes the results of the Ph.D. thesis of R. Gahleitner [4] and the model created by J.E. Berg which can be found in the final report of COST 231 [5]. Related to J.E. Berg, T. Kuerner describes his model in paper [6]. Model number seven is developed in [7] from J. Rajala. And the last model we have been looking at is from the investigation from A.M.D. Turkmani in [8].

In Table I a comparison of the features of these eight empirical models is presented where the first column denotes the reference number of the particular model. The second column shows the number of the empirical and the other parameters. It is indicated in the third column, if the model is able to distinguish between line of sight (LOS) and non line of sight (NLOS) between the base station and the external building wall. In case of this distinction, different equations are used for LOS and NLOS and thus higher accuracy can be expected.

Many empirical models need a reference value outside the building near the external wall for calculating the indoor power level. In the fourth column it is shown if the model needs this reference value outside the building in the height of each floor or only on the ground level. It is much easier to get the reference value on the ground level than in the height of each floor e.g. by performing measurements. In model [8] the calculation is not started from a reference point outside the

\footnotetext{
${ }^{1}$ There is the possibility to calculate two of the empirical via three nonempirical parameters.

${ }^{2}$ In ground floors there is always a NLOS calculation.
}

TABLE I

COMPARISON OF THE FEATURES OF EIGHT EMPIRICAL MODELS.

\begin{tabular}{|c|c|c|c|c|}
\hline Model & $\begin{array}{c}\text { Number of } \\
\text { parameters } \\
\text { emp./others }\end{array}$ & $\begin{array}{c}\text { NLOS } \\
\text { LOS }\end{array}$ & $\begin{array}{c}\text { Reference } \\
\text { at ground } \\
\text { level sufficient }\end{array}$ & $\begin{array}{c}\text { Reference } \\
\text { at Tx } \\
\text { possible }\end{array}$ \\
\hline \hline$[1]$ & $1 / 1$ & no & no & no \\
\hline$[2]$ & $3^{1}(1) / 3(6)$ & yes $^{2}$ & yes & no \\
\hline$[3]$ & $4 / 2$ & yes $^{2}$ & yes & no \\
\hline$[4]$ & $3 / 4$ & no & yes & no \\
\hline$[5]$ & $6 / 6$ & yes & yes & yes \\
\hline$[6]$ & $4 / 5$ & yes & yes & yes \\
\hline$[7]$ & $3 / 3$ & yes & no & no \\
\hline$[8]$ & $3 / 4$ & no & $/$ & yes \\
\hline
\end{tabular}

building at the external wall but at the base station antenna. Thus, there is no entry at the respective place of the table. The column "Reference at Tx possible" describes if it is possible to start the calculation directly from the base station instead of from a reference point near the building.

The advantage of [4] is that only a mean value outside the building along the external wall is necessary compared to the other models where reference values at a lot of points along the wall are required. For the calculation of the indoor power with [2] is to be taken the reference value outside the building at one wall. This is one of the disadvantages of this model because it is not always possible to define one wall as the front side, especially when more building sides do have line of sight to the base station. Other disadvantages are that the horizontal projection of the building has to be rectangular and it is not possible to calculate the indoor power near the external walls. Model [2] offers to calculate two of the three empirical parameters which results in the necessity of six non-empirical parameters instead of three. Model [3] is a further development of [2] where some disadvantages of [2] have been eliminated. For example instead of the definition of one wall as the front side, the outdoor reference values of all walls are used. The difference in [4] compared to all other models is that only one mean value along every relevant external wall is needed as outdoor reference. Furthermore, for this model it is necessary to know the heights of the floors and not only the floor level, by what it should be possible to achieve more accurate results. The advantage of [5] and [6] is the distinction between NLOS and LOS between base station and external building walls. Compared to [2], [3] and [7], which are also offering that feature, it is possible to calculate the LOS-case in all floors by starting the calculation at the transmit antenna. As [6] is related to [5] it basically offers the same features but only one mean power level for each floor is calculated. Therefore, for using [6] the number of internal walls is not required and thus one parameter less is needed. On the other hand with [6] it is not possible to calculate a mean power for one room like in model [5]. In [7] and [1] only the received power level in the ground floor can be calculated with the outdoor reference values at the ground level.

Comparing the eight empirical models we can conclude that [5] and [6] are offering the best features for predicting the indoor coverage in UMTS macro cells. Furthermore, for using these two models the highest number of parameters is 
necessary, meaning that they are expected to show the highest accuracy in different scenarios.

\section{B. Deterministic Models}

For the investigation of a deterministic model for outdoorindoor propagation in macro cells, the simulation program 'Wireless InSite' was used. This simulator uses a ray-optical method named ray-tracing [10]. In a basic ray-tracing algorithm, the main task is to determine the trajectory of a ray, launched from a transmitting antenna, including the calculation of the intersection of the rays with the objects in the propagation area. With this method reflections, transmissions, diffractions and scattering of the rays can be taken into account which leads to a higher accuracy compared to the accuracy of empirical models. Furthermore, in addition to the determination of the received power level, also the calculation of e.g. the impulse response or delay spread and the angle of arrival of the electromagnetic waves is possible. The usage of deterministic models requires the availability of the building data of the surrounding area in a very exact and maybe electronic form. In order to achieve high accuracy with ray tracing, also the exact knowledge about the electrical parameters of all the objects in the propagation path is necessary. Thus, to predict the received power level inside buildings in macro cells with deterministic models a very high effort in modeling the surroundings and a high computation power is needed.

The configuration of 'Wireless InSite' has been as follows. For the investigation of the propagation into buildings the deterministic model ' $F$ ull $3 D$ ' was used, as this model includes transmission through surfaces. As waveform for the transmitter and the receivers we used a root raised cosine like in live UMTS networks with a carrier frequency of $2140 \mathrm{MHz}$ (downlink), a pulse width of $260 \mathrm{~ns}$ and a roll-off factor of 0.22 . The simulations with imported antenna patterns for the real base station antenna in various formats (Planet, Odyssey, NSMA, XFDTD) did not show satisfactory results. Therefore, we used a simplified antenna pattern for the transmitter which describes the main lobe of the real pattern with the same beamwidths in horizontal and vertical direction. For the receivers we used isotropic antennas.

\section{InVESTIGATED SCENARIO AND MEASUREMENT SETUP}

In order to evaluate the accuracy of the models we performed measurements in a live UMTS network in the building of the Institute of Communications and Radio-Frequency Engineering at Vienna University of Technology, which is a typical office building in the city center of Vienna, Austria.

The building is made of brick and porous concrete, with a reinforced concrete frame and has large outside windows. The UMTS base station is located in a distance of $160 \mathrm{~m}$ from the institute building above the roof of a building at the height of $33.5 \mathrm{~m}$. The transmit antenna (Allgon 7520) provides a gain of $18 \mathrm{dBi}$, the electrical downtilt was set to $6^{\circ}$ with a mechanical downtilt of $0^{\circ}$. The base station was transmitting the $\mathrm{CPICH}$ at a power level of $33 \mathrm{dBm}$.
The measurements have been performed by evaluating the CPICH-RSCP (Common Pilot Channel - Received Signal Code Power) with the software tool 'TEMS Investigation WCDMA 3.0.2' from Ericsson [11] in combination with the mobile phone Motorola A835.

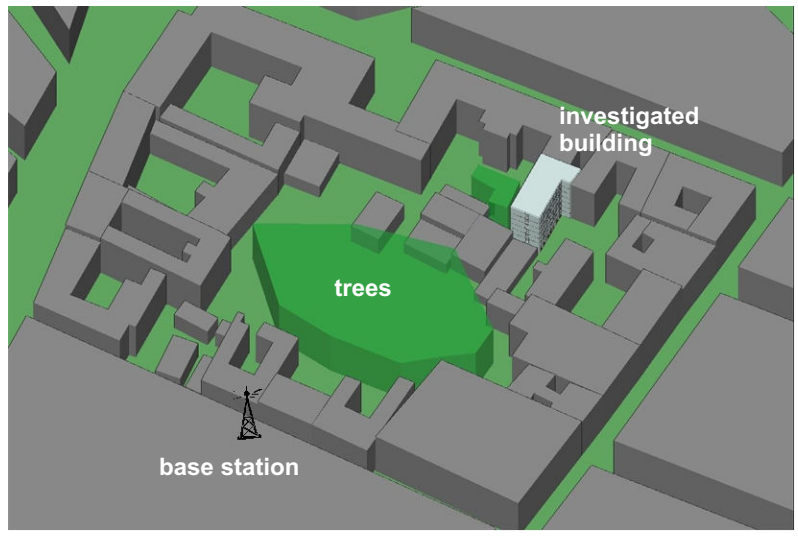

Fig. 1. Building area displayed in 'Wireless InSite'

Fig. 1 shows the investigated area as depicted in 'Wireless InSite'. For the simulations in 'Wireless InSite' the considered office building (shown in light grey) was modeled including all wall characteristics as well as the windows and floor details, whereas the surrounding buildings (dark grey) were taken as blocks with the correct heights and ground plots as they could be imported in digital form from e.g. officially available files in the AutoCAD DXF format. The dark green objects in the middle represent trees in the courtyard. Fig. 1 also shows the position of the base station of the live UMTS network which was used for measurements and simulations.

For the comparison of measurements with simulation results, measurements and simulations were performed in the corridor of each floor and in ten selected rooms in the institute building, where one mean value of the CPICH-RSCP per room or per floor was taken.

\section{COMPARison OF MOdEling- AND MEASUREMENT RESULTS}

In this section the results of the comparison between the measurements and the models (described in Section II) are presented. Table II shows the mean error and the standard deviation of the error between modeling results and measurements for each model separately - for the ground floor in column two and three, whereas in column four and five the mean error and the standard deviation is shown related to the whole building. Due to the fact that e.g. with models [1] and [7] no values for the mean error and standard deviation in the higher floors are available, whereas for the ground floor all the considered models are able to provide results, the ground floor was taken for the comparison.

Model [6] allows to calculate one mean value for each floor. Thus, there is no value for the standard deviation in the ground floor in Table II. Also in case of the simulator 'Wireless InSite' there is no value for the standard deviation in the ground floor. This is because with 'Wireless InSite' one mean value for each 
selected room was calculated and only two rooms have been investigated in the ground floor in the simulations.

TABLE II

COMPARISON BETWEEN MODELING RESULTS AND MESAUREMENTS

\begin{tabular}{|c|r|r|r|r|}
\hline \multirow{2}{*}{ Model } & \multicolumn{2}{|c|}{ Ground floor } & \multicolumn{2}{c|}{ Building } \\
\cline { 2 - 5 } & $\Delta / \mathrm{dB}$ & $\sigma_{\Delta} / \mathrm{dB}$ & $\Delta / \mathrm{dB}$ & $\sigma_{\Delta} / \mathrm{dB}$ \\
\hline \hline$[1]$ & 4.8 & 2.9 & - & - \\
\hline$[2]$ & 9.3 & 2.7 & 3.4 & 6.6 \\
\hline$[3]$ & 11.6 & 2.6 & 5.7 & 6.4 \\
\hline$[4]$ & -3.8 & 2.0 & -2.8 & 7.2 \\
\hline$[5] \mathrm{a}$ & -4.4 & 2.6 & -3.4 & 7.1 \\
\hline$[5] \mathrm{b}$ & -4.4 & 2.6 & -0.8 & 5.9 \\
\hline$[6] \mathrm{a}$ & -1.0 & - & -3.5 & 5.2 \\
\hline$[6] \mathrm{b}$ & -1.0 & - & -3.5 & 3.8 \\
\hline$[7]$ & 1.2 & 5.8 & - & - \\
\hline$[8]$ & 23.1 & 2.0 & 8.2 & 10.0 \\
\hline 'Wireless InSite' & -7.8 & - & 4.7 & 5.8 \\
\hline
\end{tabular}

Models [5] and [6] are divided into two cases. In case (a) only equations for NLOS between the base station and external building walls are used. In case (b) both NLOS-equations and LOS-equations for the higher floors are used to calculate the received indoor power levels. Model [5] provides the lowest mean error in the whole building $(-0.8 \mathrm{~dB})$ in the case of distinction between LOS and NLOS (b). Also the other results are only a little higher than the best ones. Therefore, model [5]b is the most accurate model after model [6]b, which has the lowest mean error in the ground floor $(-1.0 \mathrm{~dB})$ and the lowest standard deviation in the whole building $(3.8 \mathrm{~dB})$. As already mentioned, with models [7] and [1] it is only possible to calculate the indoor power in the ground floor. For calculating the indoor power in higher floors, outdoor reference values on the heights of each floor would be required. Additionally to this disadvantage, model [7] provides the highest standard deviation in the ground floor. Model [8] shows the highest mean error in the ground floor $(23.1 \mathrm{~dB})$ and in the whole building $(8.2 \mathrm{~dB})$ and it has the worst standard deviation in the whole building $(10.0 \mathrm{~dB})$.

When comparing the empirical models with the simulator 'Wireless InSite' by Table II, we can observe that despite the high complexity of 'Wireless InSite', the simulator was not capable of achieving results with a higher accuracy than the empirical equations.

One possibility for this high mean error $(+4.7 \mathrm{~dB})$ in the simulation results could be the fact that mobile phones have been used for measuring the received power level. There, the additional attenuation could have been caused by the person holding the mobile phone or by the differences in the evaluation of the received CPICH RSCP power level between simulations and measurements. Other reasons for the high errors of the simulations could be that the accuracy of defining the surrounding area and building characteristics has not been high enough. Especially in the modeling of the vegetation only small errors can lead to significant differences in the results. Comparing simulation results with and without the trees in the courtyard show differences in the mean received power of up to $18 \mathrm{~dB}$ in the lower floors. Also when comparing measurement results of the winter season (trees without leaves) with results of the summer season (trees with leaves), differences up to $9 \mathrm{~dB}$ can be observed.

However, in addition to having a high mean error, the results of the simulator also shows standard deviations comparable to those achieved by the empirical models.

Thus, the conclusion of this investigation is that the usage of the deterministic model is very complex, requires the availability of the (electromagnetical) characteristics of the surrounding area at a very high accuracy and since these are not available it does not provide more accurate results than simple empirical models. From this conclusion we recommend to use the empirical model [5] or model [6] for the calculation of the indoor power in urban environments. With these two models the most accurate results (see Table II) can be achieved and they are applicable in different scenarios (see Table I) while showing good usability.

\section{IDEAS FOR INDOOR COVERAGE OPTIMIZATION}

Especially in case of office buildings, where usually a lot of traffic is caused, it is of substantial interest for the network operators to optimize the indoor coverage. Due to the fact that UMTS is an interference or power limited system, the network coverage cannot be improved just by increasing the transmit power, as this would lead to less capacity in the whole network. Thus, the goal in UMTS network planning is to get optimum network coverage by using as low transmit power as possible. Ideas for optimizing the indoor coverage can be found from theoretical approaches but also from experience or results from indoor coverage prediction modeling or simulations.

In this work the conclusions from the empirical models (described in Section II) and from simulations with 'Wireless InSite' were used to find various approaches for optimizing the indoor coverage in office buildings. With the intention to achieve smallest path losses from the base station to the receivers inside the building, simulations have been performed in the scenario described in Section III, with variations of the position of the base station, the antenna heights, their downtilts and their antenna patterns. The simulation tool for cell coverage prediction ('Wireless InSite') then was used to evaluate the various ideas.

In Fig. 2 the simulated locations of the base station are shown where the black lines describe the orientation of the transmit antenna. It can be seen that the orientation of each antenna is in direction to the investigated building in order to get a reasonable comparison.

\section{A. Variation of the Antenna Height}

The path loss between the base station antenna and the indoor receiving points by varying the height of the transmit antenna is investigated in the following. The height of the antenna at location Tx01 is varied between $30 \mathrm{~m}$ and $41 \mathrm{~m}$. The downtilt of the transmit antenna in all the simulations is set in a way that the orientation of the antenna is towards the center of the external wall of the considered building. 


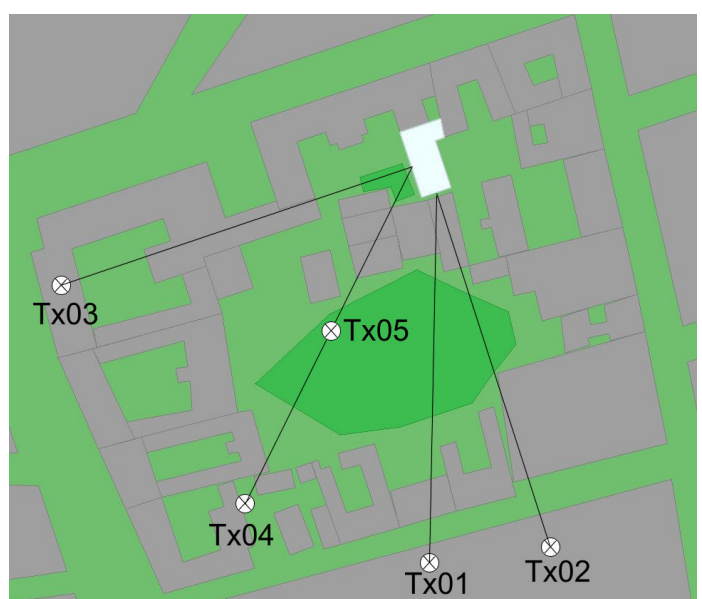

Fig. 2. Location and orientation of the (virtual) base station antennas.

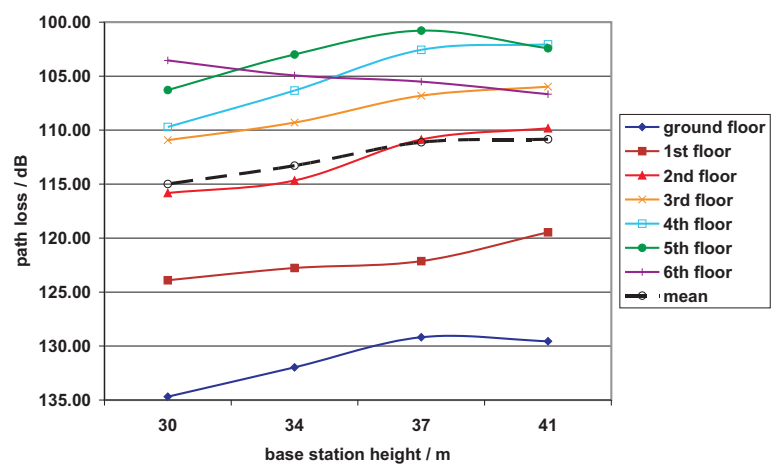

Fig. 3. Mean path loss vs. different transmit antenna heights.

In Fig. 3 the mean path loss for each floor and for the whole building is shown vs. the height of the antenna. We can see that the path loss is decreasing with increasing height of the transmit antenna for almost all floors. Only the results for the 6th floor are showing a contrary trend which can be due to the permanent LOS connection in the 6th floor. Considering the mean value over the whole building a trend to lower path losses at higher antenna heights can be observed. Similar results can be shown for the other locations.

\section{B. Variation of the Antenna Position and the Downtilt}

For the positions Tx01-Tx04 (see Fig. 2) we have investigated the impact of the variation of the location and the downtilt of the antenna on the path loss. At two of the selected locations $(\mathrm{Tx} 02, \mathrm{Tx} 03)$ the electromagnetic waves are arriving at an angle of $90^{\circ}$ at the external wall of the investigated building, whereas at location Tx04 the base station antenna is in a LOS position for two building sides with an angle of incidence of $45^{\circ}$ at both walls. At all four locations the height of the transmit antenna was set to $33.5 \mathrm{~m}$.

As expected, Fig. 4 shows that the optimum antenna downtilt for reaching minimum mean path loss is different for each floor, meaning that it is optimum to point the main beam of the antenna directly to the respective floor or to adjust the main beam to $2 / 3$ ( $6^{\circ}$ downtilt) of the height of the building for reaching minimum mean path loss in the whole building.

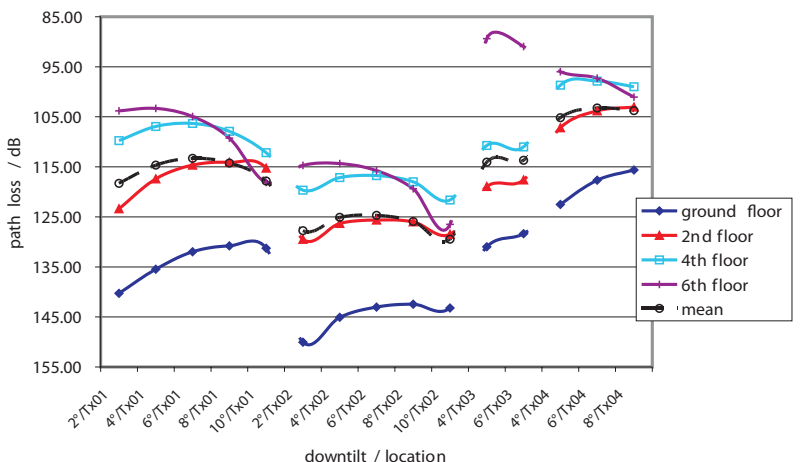

Fig. 4. Mean path loss vs. different locations and downtilts of the transmit antenna

These results tend to be the same for all locations.

When comparing the results at the different locations it can be observed that the smallest mean path loss could be achieved at position Tx04. This is the position where two building sides are in line of sight to the base station antenna. That result is in contradiction to some modeling approaches (e.g. in [5]) where the lowest path loss is predicted in case of an angle of arrival of $90^{\circ}$ at the building wall.

\section{Variation of the Antenna Pattern}

The investigation of the impact of the antenna patterns on the mean path loss has been performed at all the antenna positions as depicted in Fig. 2 with an antenna height of $33.5 \mathrm{~m}$ and the antenna downtilt was set to $6^{\circ}$ for Tx01-Tx04. At position Tx05 an isotropic antenna (Antenna V) was used. Table III shows the characteristics of the different antennas in terms of half power beamwidth vertical $\left(\theta_{3 d B, V}\right)$ and horizontal $\left(\theta_{3 d B, H}\right)$. In order to keep the results comparable, $6 \mathrm{~dB}$ was added to the path loss measured with the base station antenna at $\mathrm{Tx} 05$ since the distance of the base station position Tx05 to the building is only the half compared to all the other positions.

TABLE III

CHARACTERISTICS OF THE USED ANTENNAS

\begin{tabular}{|c||c|c|c|c|}
\hline & Ant I & Ant II & Ant III & Ant IV \\
\hline$\theta_{3 d B, V}$ & $6.5^{\circ}$ & $13^{\circ}$ & $6.5^{\circ}$ & $6.5^{\circ}$ \\
\hline$\theta_{3 d B, H}$ & $65^{\circ}$ & $65^{\circ}$ & $32^{\circ}$ & $16^{\circ}$ \\
\hline
\end{tabular}

In Fig. 5 the mean path loss simulated with different transmit antenna patterns and at different locations of the base station antenna is presented. As already observed in Fig. 4, again we can conclude from Fig. 5 that in general the mean path loss is less if there is a LOS connection between the base station antenna and two building sides. We can see that a minimum for the mean value of the whole building and for all the floors except the 6th floor can be reached, when transmitting from positions $\mathrm{Tx} 04$ or $\mathrm{Tx} 05$.

Concerning the different transmit antenna patterns at the base station we can observe that a minimum mean path loss can be reached when using the antenna with the largest beamwidths at any location.

One possible explanation is presented via Fig. 6 where the propagation paths from the transmit antenna into the corridor of the ground floor are shown when using Antenna I and Antenna III (smaller horizontal beamwidth) at position 


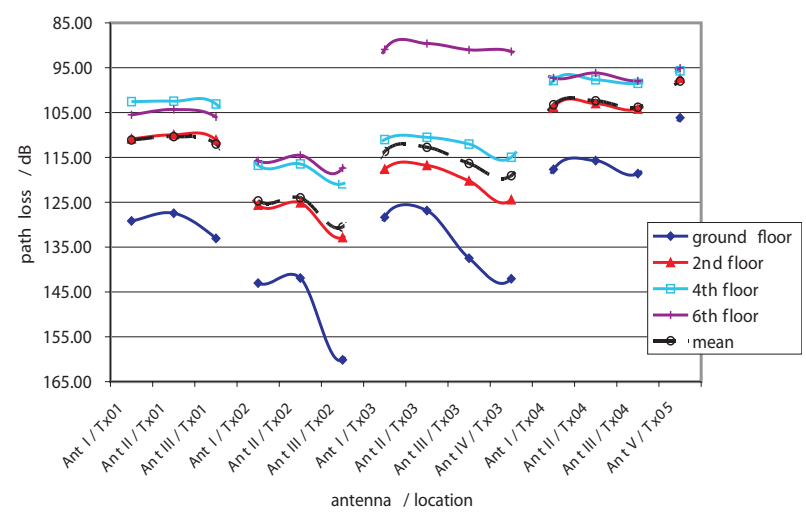

Fig. 5. Mean path loss vs. different locations and antenna patterns.

Tx03. It can be seen that in both cases the propagation paths near the main direction of the antenna are approximately the same. But due to the larger beamwith in case of Antenna I, additional paths at larger angles of departure are becoming more important as they are carrying significant energy to the receiving points in the ground floor of the building.

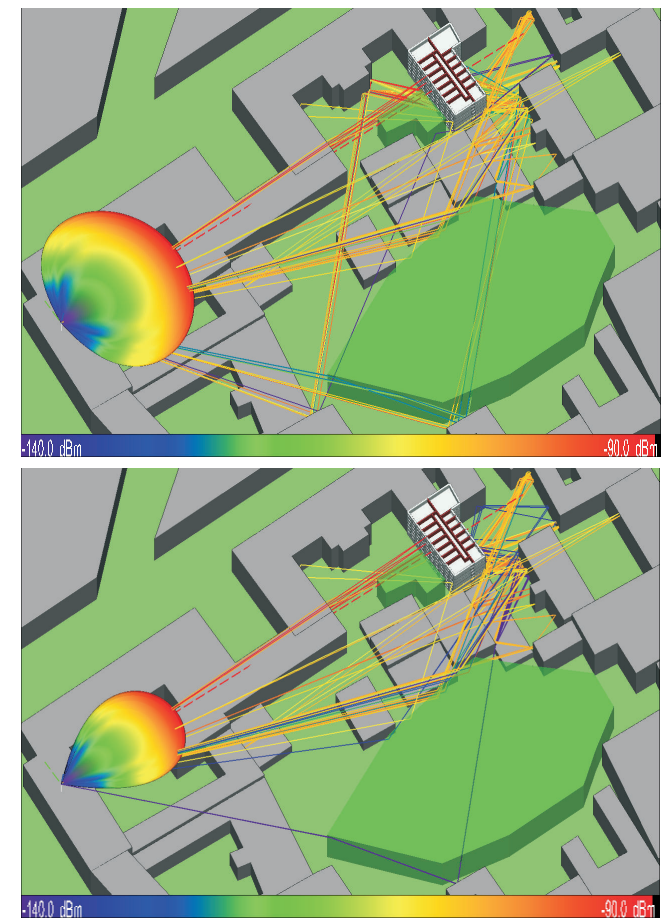

Fig. 6. Propagation paths into the corridor of the ground floor with Antenna I (upper picture) and Antenna III (lower picture).

\section{SUMmary AND CONCLUSIONS}

We present a comparison between eight empirical and one deterministic model (ray tracing) for the prediction of indoor coverage in UMTS macro cells. The evaluation of the models was done regarding usability, general validity and accuracy of the models. For the evaluation of the accuracy of the models, the modeling results have been compared to results from measurements, performed in an office building in a UMTS macro cell in the city center of Vienna, Austria.

Despite the high effort in modeling the (electromagnetical) characteristics of the surrounding area and the high computation time for using the deterministic model within the simulator, no higher accuracy could be reached with the deterministic model compared to the empirical models.

As for predicting the indoor coverage, mobile operators need a simple model with a high usability and high accuracy at the same time, we recommend to use the empirical models [5] or [6] for the calculation of the outdoor-indoor propagation in urban environments. With these two models the most accurate results have been achieved and they are applicable in different scenarios while showing good usability.

In the last section of this document the evaluation of various ideas for the optimization of the indoor coverage in urban environment is presented. For improving the outdoor-indoor propagation in UMTS macro cells the base station antenna position, the antenna height, its downtilt and antenna patterns are varied. Results show that indoor coverage can be improved with higher base station antennas, and also when having a position for LOS to two building sides. Furthermore, it was shown that better indoor coverage can be reached in dense urban environments when using antenna patterns with larger beamwidths which open up more radio paths.

\section{ACKNOWLEDGEMENTS}

This document is based on the master's thesis [12] of A. Paier at Vienna University of Technology, Austria. We thank mobilkom austria AG\&CoKG for technical and financial support of this work. The views expressed in this paper are those of the authors and do not necessarily reflect the views within mobilkom austria.

\section{REFERENCES}

[1] R. Hoppe, G. Woelfle, F.M. Landstorfer, "Measurement of building penetration loss and propagation models for radio transmission into buildings," 50th IEEE Vehicular Technology Conference, Amsterdam, The Netherlands, pp. 2298-2302, September 1999.

[2] Z. Liu, L. Slawitschka, G. Zgunea, "Measurements and Modelling of Building Penetration Loss at $900 \mathrm{MHz}$," Publication of DeTeMobil, Bonn, October 1993.

[3] http://www.inue.uni-stuttgart.de/FMS/abschluss/ berichte/fms213-03.pdf

Meilensteinbericht: "Ausbreitungsmodelle zur Berechnung der Feldstärkeversorgung in Gebäuden bei gegebener Aussenversorgung", 1998.

[4] R. Gahleitner, "Radio Wave Propagation in and into Urban Buildings," Ph.D. Thesis, Vienna University of Technology, Austria, 1994.

[5] J.E. Berg, "Building Penetration," in Digital Mobile Radio Toward Future Generation Systems (COST 231 Final Report), Brussels, Belgium: COST Telecom Secretariat, CEC, sec. 4.6, pp. 167-174, 1999.

[6] T. Kuerner, A. Meier, "Prediction of Outdoor and Outdoor-to-Indoor Coverage in Urban Areas at $1.8 \mathrm{GHz}$," Selected Areas in Communications, IEEE Journal on, vol. 20, no. 3, pp. 496-506, April 2002.

[7] J. Rajala, K. Sipilae, K. Heiska, "Predicting In-Building Coverage for Microcells and Small Macrocells," 49th IEEE Vehicular Technology Conference, Houston, USA, pp. 180-184, 1999.

[8] A.M.D. Turkmani, A.F. de Toledo, "Modelling of Radio Transmission into and within Multistorey Buildings at 900, 1800 and $2300 \mathrm{MHz}$," IEE Proc.- Part I, vol. 140, no. 6, pp. 462-470, December 1993.

[9] http://www.remcom.com/WirelessInSite/index.html

[10] R. Vaughan, Channels, Propagation and Antennas for Mobile Communications, Institute of Electrical Engineers, London, 2003.

[11] http://www.ericsson.com/products/hp/TEMS \ _Products $\backslash$ _pa.shtml

[12] A. Paier, "Berechnung und Optimierung der UMTS Netzabdeckung innerhalb von Gebäuden," Master's Thesis, Vienna University of Technology, Austria, Feb. 2006. 